\title{
The topology and polarisation of subbeams associated with the "drifting" subpulse emission of pulsar B0943+10
}

\section{Q-to-B-mode recovery dynamics}

\author{
Joanna M. Rankin ${ }^{1, \star}$ and Svetlana A. Suleymanova ${ }^{2,3}$ \\ 1 Sterrenkundig Instituut "Anton Pannekoek", 1098 SJ Amsterdam, The Netherlands \\ e-mail: jrankin@astro.uva.nl \\ 2 Pushchino Radio Astronomy Observatory, 142292 Pushchino, Russia \\ e-mail: suleym@prao.psn.ru \\ 3 Isaac Newton Institute of Chile, Pushchino Branch, Russia
}

Received 1 September 2005 / Accepted 20 March 2006

\section{ABSTRACT}

\begin{abstract}
Pulsar B0943+10 is well known for its "B" (burst) mode, characterized by accurately drifting subpulses, in contrast to its chaotic "Q" (quiet) mode. Six new Arecibo observations at $327 \mathrm{MHz}$ with durations of $2+$ h each have shed considerable light on the modal dynamics of this pulsar. Of these, three were found to be exclusively " $\mathrm{B}$ " mode, and three were discovered to exhibit transitions from the "Q" to the "B" mode. One of these observations has permitted us to determine the circulation time of the subbeam carousel in the "Q" mode for the first time, at some $36.4 \pm 0.9$ stellar rotation periods. The onset of the "B" mode is then observed to commence similarly in all three observations. The initial circulation time is about 36 periods and relaxes to nearly 38 periods in a roughly exponential fashion with a characteristic time of some $1.2 \mathrm{~h}$. This is the longest characteristic time ever found in a mode-switching pulsar. Moreover, just after the "B"-mode onset the pulsar exhibits a symmetrical resolved-double profile form with a somewhat stronger trailing component, but this second component slowly dies away leaving the usual single "B"-mode profile with the longitude of the magnetic axis falling at about its trailing half power point. Thus it would appear that Q-to-B- and B-to-Q-transitions have different characteristic times. Some speculations are given on the nature of this slow modal alternation.
\end{abstract}

Key words. magnetohydrodynamics (MHD) - plasmas - pulsars: general - pulsars: individual: B0943+10 radiation mechanism: nonthermal - polarization

\section{Introduction}

Pulsars are known to form very stable intensity profiles after averaging hundreds of individual pulses. Additionally, there are a number of peculiar, so-called "mode switching" pulsars, which have two stable integrated-profile shapes. One prominent such pulsar is B0943+10. Several studies have described the emission characteristics of its bright "B" mode and less intensive (in average) "Q" mode (Suleymanova \& Izvekova 1984; Suleymanova et al. 1998, hereafter SIRR).

In 1992 a nearly 1000-pulse sequence was recorded using the Arecibo radio telescope at $430 \mathrm{MHz}$, and it was found to begin with some $800+$ pulses in the B mode followed by nearly 200 pulses in its Q mode. The transformation process from the $\mathrm{B}$ to $\mathrm{Q}$ mode was thoroughly investigated. It was shown that the Q-mode onset resulted from both abrupt and gradual changes in the intensity of individual pulses. The presence of slow changes in the modal transformation process having a timescale of some 20 min were documented for the first time.

This key Arecibo observation was also used to determine the subbeam configuration responsible for the driftingsubpulse pattern for the first time (Deshpande \& Rankin 1999, 2001, hereafter Paper I), and 35-MHz Gauribidanur observations revealed the same B-mode pattern at very low frequency

^ On leave from Physics Dept., University of Vermont, Burlington, VT 05405 USA; e-mail: joanna.rankin@uvm. edu
(Asgekar \& Deshpande 2001, hereafter Paper II). Furthermore, a remarkable set of simultaneous $40-$ and $103-\mathrm{MHz}$ recordings made at the Pushchino Radio Astronomy Observatory suggested interesting correlations between the circulation time, profile form and emission mode (Rankin et al. 2003, hereafter Paper III), but the short duration of these pulse sequences (hereafter PSs) from transit instruments left many questions unanswered about B0943+10's overall modal dynamics.

It is thus of great interest to further investigate the star's modal transformation processes. Observations of B0943+10 were carried out using the upgraded Arecibo radio telescope in 2003 at $327 \mathrm{MHz}$ with this aim. As a result, several individual pulse observations have been recorded with durations up to $135 \mathrm{~min}$. Remarkably, three of the six observations contain PSs of both modes, all transitions from the $\mathrm{Q}$ to the $\mathrm{B}$ mode. One of our main questions was whether the transition process between the star's two modes is symmetrical. Having heretofore had access to only two short records with modal transitions (B-Q at $430 \mathrm{MHz}$ in Paper I) and (Q-B at 103/40 MHz in Paper III), we now have an excellent opportunity to answer to this question. Section 2 discusses our observations, Sect. 3 compares the properties of the two modes, and Sect. 4 discusses the profile variations incurred in the mode change. Fluctuation-spectral variations associated with the modal transition are treated in Sect. 5, and in Sect. 6 we attempt to understand where our other B-mode sequences would fall in relation to the modal onset. Section 7 
Table 1. Arecibo 2003 observations at $327 \mathrm{MHz}$.

\begin{tabular}{cccccc}
\hline \hline Day & MJD & 2003 & Mode & $\begin{array}{c}\text { Switch } \\
\text { (pulse) }\end{array}$ & $\begin{array}{c}\text { Length } \\
\text { (pulses) }\end{array}$ \\
\hline 1 & 52709 & 10 March & Q to B & 2540 & 6748 \\
2 & 52711 & 12 March & B & - & 6809 \\
3 & 52832 & 12 July & Q to B & 5266 & 7559 \\
4 & 52840 & 20 July & B & - & 7274 \\
5 & 52916 & 4 October & Q to B & 1755 & 5650 \\
6 & 52917 & 5 October & B & - & 3024 \\
\hline
\end{tabular}

discusses the asymmetry between the B-to-Q and Q-to-B transitions processes, and Sects. 8 and 9 give brief discussions and summaries of the results, respectively.

\section{Observations}

The observations used in our analyses were made using the 305-m Arecibo Telescope in Puerto Rico together with its Gregorian reflector system and new 327-MHz polarimetric receiver system. The signals were acquired using the Wideband Arecibo Pulsar Processor (WAPP ${ }^{1}$ ) during 2003 as detailed in Table 1. The auto- and cross-correlation functions of the channel voltages produced by receivers connected to orthogonal linearly polarized feeds were 3-level or 9-level (days 52916/7) sampled. Upon Fourier transforming, 64 or more channels were synthesized across a $25-\mathrm{MHz}$ bandpass with a $512-\mu$ s sampling time, providing a resolution of less than a milliperiod. The Stokes parameters have been corrected for dispersion, interstellar Faraday rotation, and various instrumental polarization effects ${ }^{2}$.

\section{Characteristics of B0943+10's B and Q modes}

Polarized 327-MHz integrated profiles of B0943+10's B and $\mathrm{Q}$ modes are given in Figs. 1 and 2. These observations were carried out on 2003 October 5 and 4, respectively. The top panels give the total intensity (solid), linear (dashed) and circular (dotted curve) polarization, and the lower panels position-angle (hereafter PA) histograms. Table 2 summarizes some of the profile properties.

The peak intensity of the $Q$ mode is typically $1 / 3$ that of its B-mode; however the Q-mode profile is roughly twice as wide as its B-mode counterpart. The second component of the "pure B"-mode profile is unresolved at $327 \mathrm{MHz}$ - similar to the situation at $430 \mathrm{MHz}$ (e.g., Paper I) but contrasting with that at $103 \mathrm{MHz}$, where the second component is $1 / 3$ of the amplitude of main component and well resolved, its peak trailing at a distance of $10^{\circ}$ (SIRR).

The lower panels in Figs. 1 and 2 show the primary- and secondary-polarization modes (hereafter PPM and SPM), which form two bands of PA sweep in longitude, separated by $90^{\circ}$. The B mode is dominated by the PPM. The PA distribution in Fig. 2 is typical for the Q mode: the PPM (lower band) and SPM (upper band) span the entire longitude range under the pulse window and the sample PAs are almost equally distributed between the two modal bands, resulting in a nearly complete depolarization of the integrated profile.

\footnotetext{
1 http://www . naic. edu/ wapp

2 Unfortunately, an error in the WAPP software resulted in only one linear polarization being recorded, four-fold redundant, during the initial 2003 March observations.
}
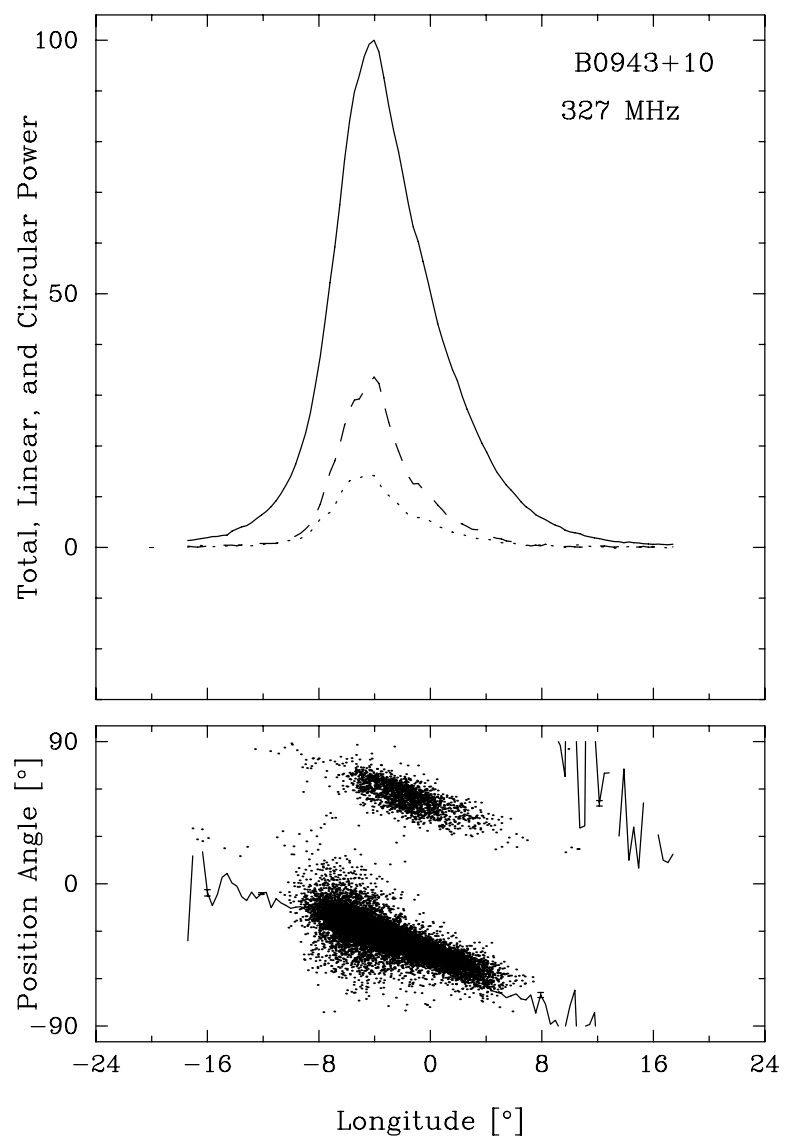

Fig. 1. Average polarization profile giving the total intensity (solid line), total linear (dashed line) and circular polarization (dotted line) for the B-mode of PSR B0943+10 (top panel). Linear polarization angles for the integrated profile (solid line) and sufficiently strong individual pulse samples (dots) are given as a function of pulse longitude (bottom panel). The 327-MHz PS was recorded on 2003 October 5.

\section{The Q- to B-mode transitions of $B 0943+10$ at $327 \mathrm{MHz}$}

We first discovered the Q- to B-mode transition of 2003 March 10, and the intensity and profile changes associated with this event are shown in Figs. 3 and 4. The central panel of Fig. 3 gives a set of 482-pulse averages, five before the transition and nine afterward. The transition occurred at about pulse 2540 as we will discuss further below. Note the initial weak, broad, unimodal Q-mode profile which then gives way to a double B-mode form having a more intense trailing component. This unusual trailing component then weakens progressively over the next hour or so, finally resulting in the usual B-mode profile seen in Fig. 1 - that is, with an asymmetrical single form that has longitude zero (that of the magnetic axis) near its trailing half-power point. The left-hand panel gives the integrated intensity of each average, and it is clear that the B-mode onset entails an about 50\% increase in the star's intensity. Figure 4 gives the same information but in averages of only 27 pulses. The breadth of the disorderly Q-mode emission is very clear in this figure as is the brightness of the occasional intense Q-mode pulses which contribute to the averages that are plotted. The two bright features between pulses 5500 and 6000 are probably caused by interference. The lower panels in each figure give the profile computed over the full length of the observation and are thus identical. Note that this profile is double attesting to the strength of the transient 

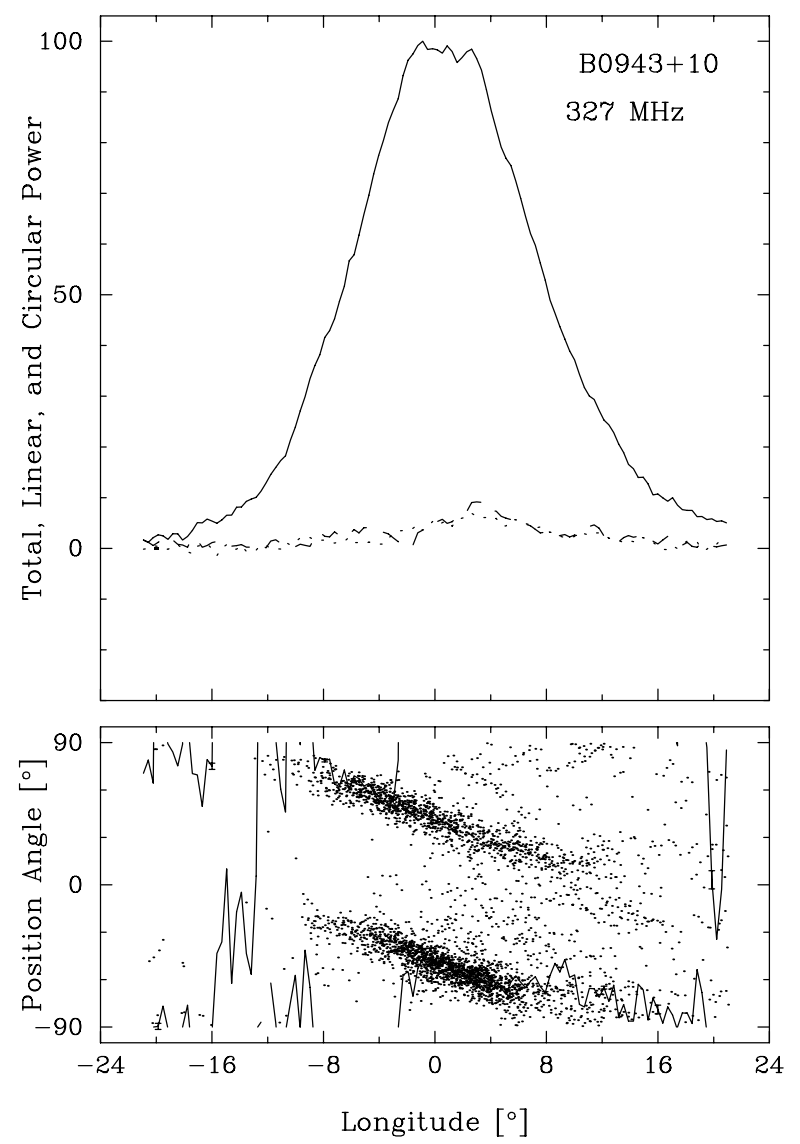

Fig. 2. Average polarization profile and polarization-angle distribution as in Fig. 1 for the Q-mode of PSR B0943+10. This 327-MHz observation was recorded on 2003 October 4.

Table 2. Modal profile properties of B0943+10 at $327 \mathrm{MHz}$.

\begin{tabular}{cccccc}
\hline \hline Mode & $\begin{array}{c}\text { Flux } \\
(\text { arb. })\end{array}$ & $\begin{array}{c}W \\
(\mathrm{~ms})\end{array}$ & $\begin{array}{c}W \\
\left({ }^{\circ}\right)\end{array}$ & $\begin{array}{c}L / I \\
(\%)\end{array}$ & $\begin{array}{c}V / I \\
(\%)\end{array}$ \\
\hline B & $2 ?$ & 20 & 7 & 30 & 15 \\
Q & 1 & 41 & 14 & 10 & 10 \\
\hline
\end{tabular}

second component for a substantial period after the modal transition.

Remarkably, the two other Q- to B-mode transitions identified in our observations behave very similarly. The one on 12 July occurred near pulse 5266 rather late in that day's observation, and a higher level of interference on 4 October initially complicated our finding the event there at pulse 1755 . The left-hand portion of Fig. 5 shows how the profile changes in all three transitions track each other. Here we plot the ratio of the component-height maxima $A(2 / 1)$ as a function of profile number, where each profile consists of 256 successive pulses. The three transitions are aligned such that profile 1 of each begins with its first B-mode pulse. Note how closely the three first profiles resemble each other, all with their second component peak amplitudes a remarkable 1.75 times their first! The diagram shows considerable scatter within the first 15 or so profile amplitude ratios, but overall one has the impression of an exponential-like behavior. And it is useful to keep in mind that profile 15 follows the B-mode onset by some $70 \mathrm{~min}$ !

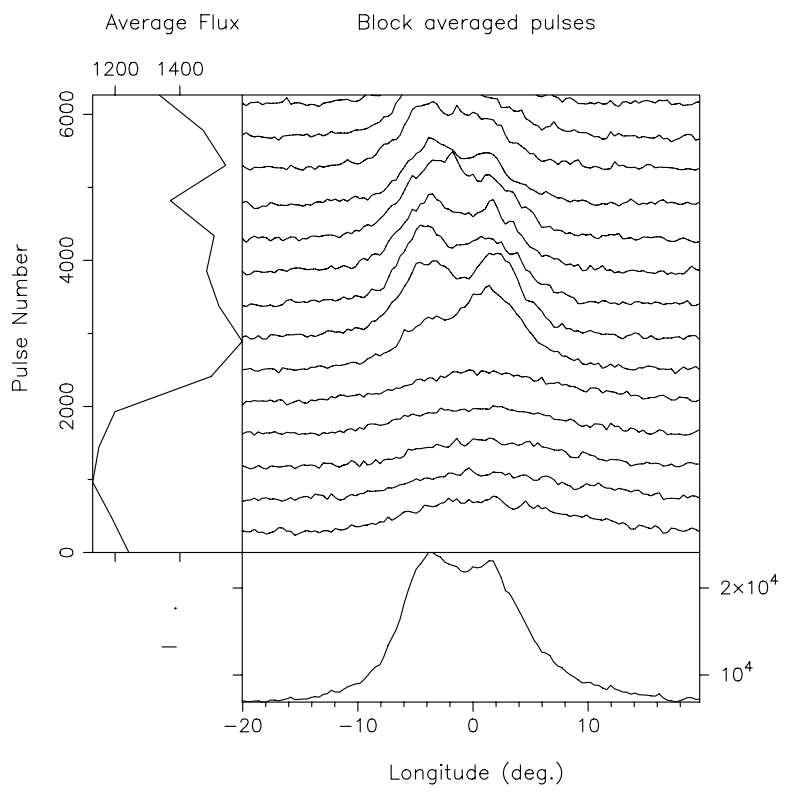

Fig. 3. A set of total intensity pulse profiles of B0943+10 at $327 \mathrm{MHz}$ (2003 March 10), obtained by integration of 482 consecutive pulses. The first 5 integrations show the smooth, broad and weak profiles of the Q-mode. Note the unusual profile shape just after the burst-like Bmode onset with the strongest emission coming after the magnetic-axis (zero) longitude. The subsequent profiles show the gradually decreasing intensity of the second component. The broad shape of the total integration in the bottom panel shows that Q-like features persist well after the B-mode onset. The intensity integrated over the pulse window as a function of pulse number is shown in the left-hand panel.

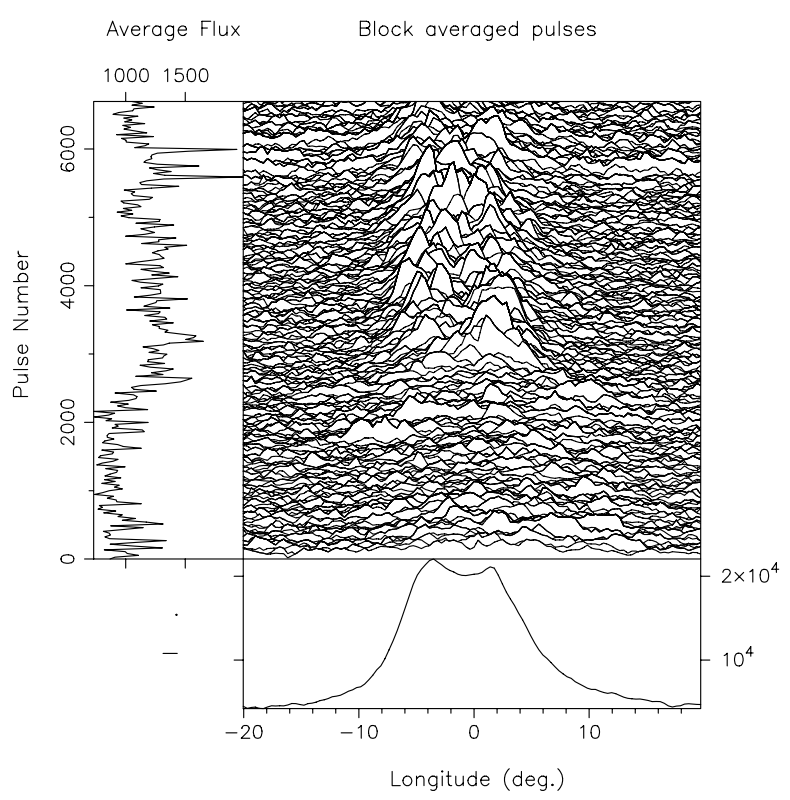

Fig. 4. Another set of profiles of the B0943+10 sequence in Fig. 3, obtained here by integration of 27 pulses. The abrupt onset of the B mode at about pulse 2540 is associated with a $50 \%$ increase in the emission intensity and the resumption of subpulse drifting behavior.

\section{Variation of the "carousel" circulation time $\hat{\mathbf{P}}_{3}$}

In Paper III we noted interesting correlations between the forms of the B-mode profiles and the circulation times computed from fluctuation spectra of their constituent PSs. The various observations in that paper, though extensive, were of inadequate length to see how these variations came about. Moreover, it was 


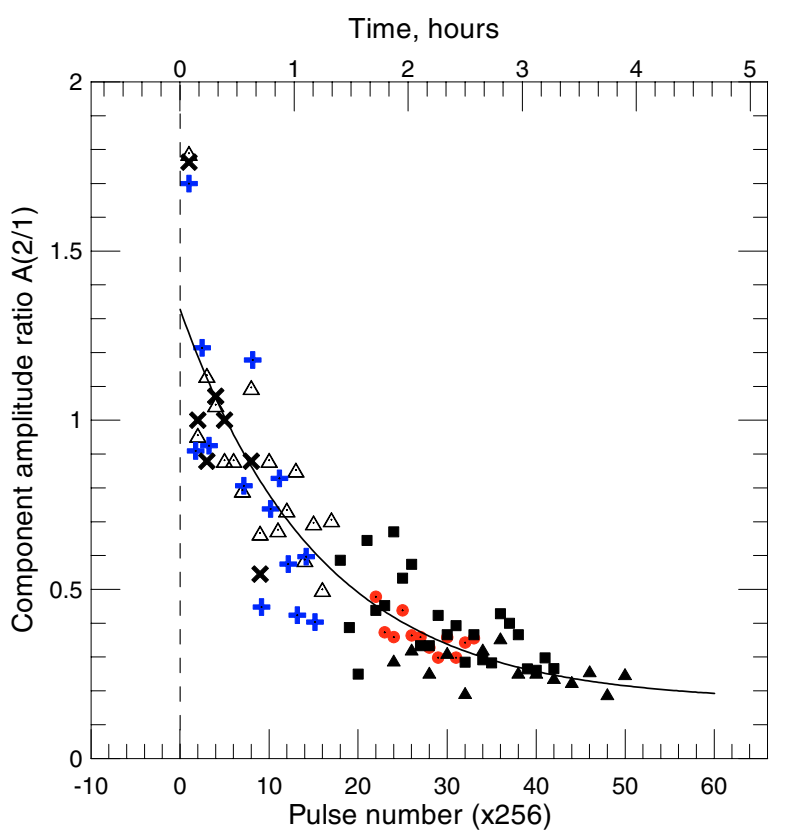

Fig. 5. Systematic changes in B0943+10's average-profile form as a function of time after the B-mode onset. Initially the trailing component dominates, but its profile slowly evolves to its usual asymptotic form, where the longitude of the magnetic axis falls near the trailing half-power point and the intensity of the second component decreases to $1 / 5$ that the first, dominant component. Here, we plot the amplitude ratio $A(2 / 1)$ of the two component peaks, separated by $6^{\circ}$ longitude, as a function of time in terms of the profile number. Each average is comprised of 256 individual pulses, or $280 \mathrm{~s}$ ( $4.7 \mathrm{~min}$ ). Note the remarkable similarity of the very first B-mode pulse shape with $A(2 / 1)$ near 1.75 for all three Q- to B-mode transition days (hereafter MJD 52709 open triangles, $52832 \mathrm{Xs}, 52916$ crosses). Values for the "pure B" days (MJD 52711 squares, 52840 triangles and 52917 circles) are shifted to begin at profile numbers 18,24 and 22 , respectively. They clearly show a gradual change of pulse shape and seem to represent a later portion of the Q- to B transformation process (see also Fig. 10). This interpretation is further supported by the gradually increasing circulation time of all three "pure B" days (see text and Fig. 8). The vertical dotted line indicates the B-mode onset time. A fit to the values (solid curve) has the form $0.17+1.16 \exp (-t / \tau)$, where $\tau$ is $15.65256-P_{1}$ blocks.

unclear whether subbeam circulation persisted in the Q mode; we found some hints in Paper I that it did do, but no Q-mode PS then in our possession exhibited a fluctuation feature demonstrating such circulation.

We were thus surprised and gratified when the Q-mode PS preceding the B-mode onset of 2003 March 10 exhibited a strong low frequency modulation feature. The longituderesolved fluctuation (hereafter lrf) spectra are given in Fig. 6. They are computed from the first 2048 pulses in the PS having a B-mode onset at pulse 2540. A "boxcar" smoothing of 3 samples has been applied to the PS to enhance the $\mathrm{S} / \mathrm{N}$ of the spectra. The $\mathrm{Q}$-mode profile is clearly recognizable in the left-hand panel and the integral spectrum is shown in the lower panel where a strong feature at some 0.025 cycles/period (hereafter $\mathrm{c} / P_{1}$ ) is evident. Careful measurement of the frequency of this feature results in a value of $0.0275 \pm 0.001 \mathrm{c} / P_{1}$. This is the first time that a fluctuation feature corresponding to the circulation time has been seen in our meter-wavelength observations, though such a feature has at times been seen in the decameter PSs of Paper II. The circulation time associated with this fluctuation frequency is then some $36.4 \pm 0.9 P_{1}-$ a somewhat lower value that usually found for B-mode circulation times.

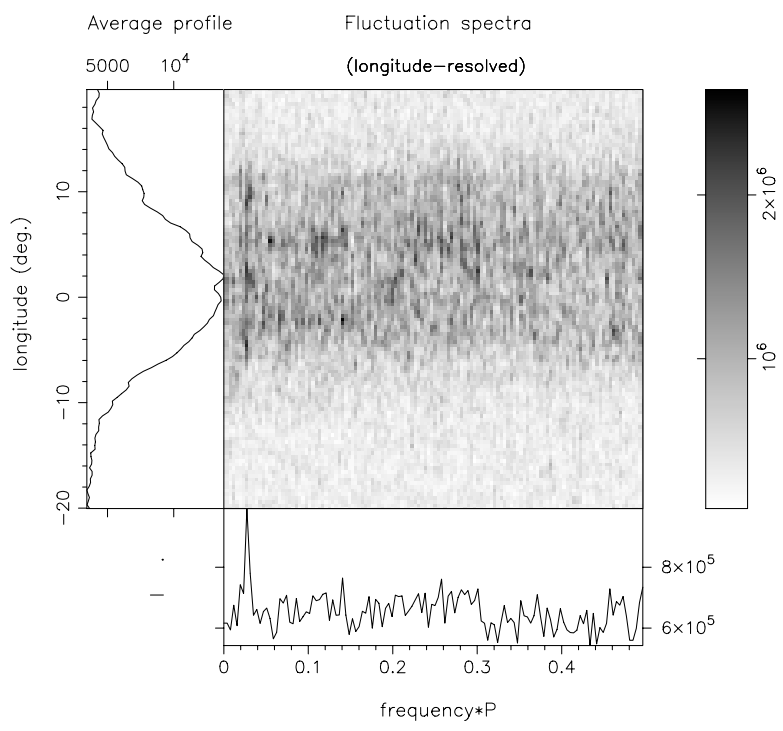

Fig. 6. Longitude-resolved fluctuation spectra for the initial Q-mode interval of the 2003 March 10 observation. The prominent feature in the bottom panel at $0.0275 \pm 0.001 \mathrm{c} / P_{1}$ corresponds to an interval of $36.4 \pm 0.9 P_{1}-$ apparently the first detection of a Q-mode circulation time. Note also the complete lack of a B-mode "drift" feature at about $0.45 \mathrm{c} / P_{1}$ and the broad, single Q-mode profile centered at zero longitude. These MJD 52709 spectra were computed using the first 2048 pulses, whereas the B-mode recommences at about pulse 2540 .

Subbeam circulation times can more readily be computed for B-mode PSs from the strong roughly $0.46 \mathrm{c} / P_{1}$ subpulsemodulation feature always associated with this behavior. We further know that this response is a first-order alias of the true fluctuation frequency of about $0.54 \mathrm{c} / P_{1}-$ a matter which has been demonstrated extensively using the harmonic-resolved fluctuation spectrum (e.g., Paper I). Finally, every analysis of the star's B-mode properties has resulted in the conclusion that the observed subpulse drift is produced by a circulating pattern of just 20 subbeams. Thus, the B-mode circulation time can be computed as $\hat{P}_{3}=20 P_{3}$ (true) $=20 /\left[1-f_{3}(\right.$ obs $\left.)\right]$.

We give an example of such a computation in Fig. 7 for the 256 pulses immediately after the B-mode onset of the 2003 March 10 observation at pulse 2540. Here the highly unusual profile just following the B-mode onset is given in the left-hand panel, and the bottom panel shows the integral spectrum associated with the drifting-subpulse modulation. The feature frequency is here some $0.452 \pm 0.003 \mathrm{c} / P_{1}-$ one of the smallest such values ever measured for the star's B mode.

Generally, it was possible to measure a circulation time for each 256-pulse interval following the respective B-mode onsets in each of the three observations. Not only do the $\hat{P}_{3}$ values of these three transitions behave similarly as a function of time, but they too suggest an exponential dependence of the form $[1-\exp (-t / \tau)]$. The behavior of all three observations is plotted in the left-hand part of Fig. 8, where the three observations are depicted with triangle, + and $\times$ symbols. Note as well that several other of the initial circulation-time values fall at just more than $36 P_{1}$, thus agreeing well with the more poorly determined Q-mode value from Fig. 6.

Remarkably, we can then apparently conclude that the initial B-mode, subpulse-drift associated circulation time represents a continuation of that associated with the very disorderly emission of the star's Q mode. 


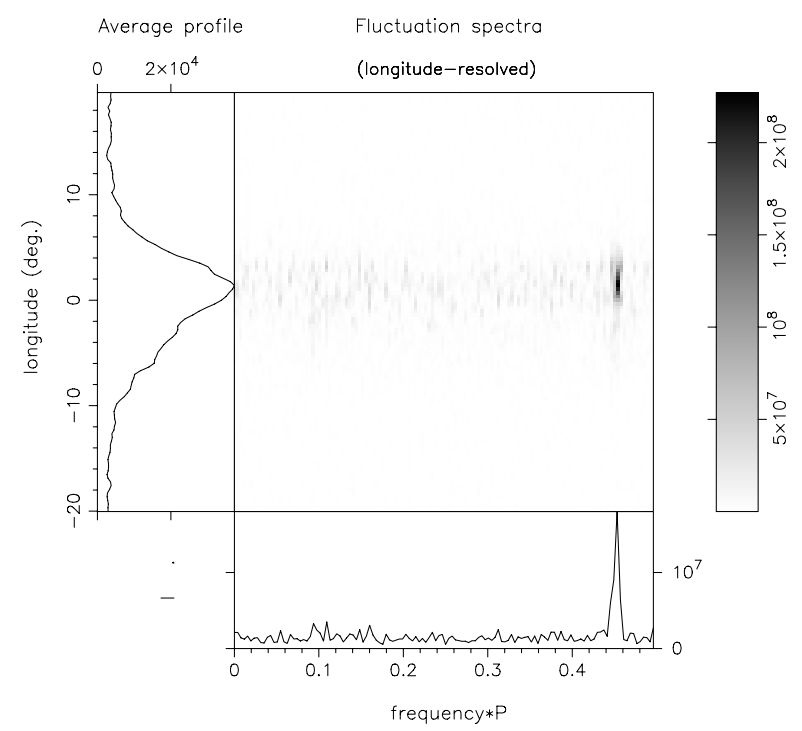

Fig. 7. Longitude-resolved fluctuation spectra computed from 256 pulses just after the B-mode onset of the 2003 March $10 \mathrm{ob}-$ servation. Here the "drift" feature frequency is $0.452 \mathrm{c} / P_{1}$, which corresponds to a 20-subbeam "carousel" circulation time of $36.47 \pm 0.03 P_{1}$ - just the same as that determined for the Q-mode in Fig. 6.

\section{Locating the "pure B"-mode observations within the transition recovery}

We then turned our attention to the analysis of the three long PSs in which only B-mode emission was observed, those recorded on 12 March, 20 July and 5 October, respectively. Each PS exhibited two properties which surprised us initially: a) there was a perceptible tendency for the amplitude ratio $A(2 / 1)$ to decrease; and b) we also found strong evidence in two of the three for a lengthening of the circulation time over their duration. Of course, we now understand that a Q- to B-mode transition had to occur somewhat before the beginning of our data-acquisition in each of these cases - the only question is just how long before.

The profile and circulation-time variations give us an indication of just where the "pure B"-mode observations fall in relation to the B-mode onset. If we proceed on the strong appearance that these quantities do exhibit a $[1-\exp (-t / \tau)]$ functional dependence, then we can locate the various average profiles and their corresponding $\hat{P}_{3}$ values appropriately and then partially test whether the procedure was correct. Indeed, we find again that the two measures vary together such that the three days can be interpolated at a time corresponding to the 18th, 24th and 22nd 256-pulse average after the B-mode onset, respectively. We have plotted the $A(2 / 1)$ values in Fig. 5 and the $\hat{P}_{3}$ measurements in Fig. 8 as is also described in their captions.

While the above interpolation procedure must be regarded as approximate, it also cannot be wildly wrong. The above circulation-time values following the B-mode onset were fitted to a $[1-\exp (-t / \tau)]$ function, which is shown by the continuous curve in Fig. 8. The characteristic time $\tau$ is then about $73 \mathrm{~min}$ or $4000 P_{1}$. It is also some 107 circulation times. In terms of the light-time scale of the pulsar's magnetosphere, to say nothing of the acceleration regions within its polar flux tube, this is a very long time indeed. It is hard to imagine what physical processes associated with pulsar emission could have such a long time scale.

Finally, in Fig. 9 we plot the component peak-amplitude ratio $A(1 / 2)$ versus the subbeam-circulation time $\hat{P}_{3}$ for all of six

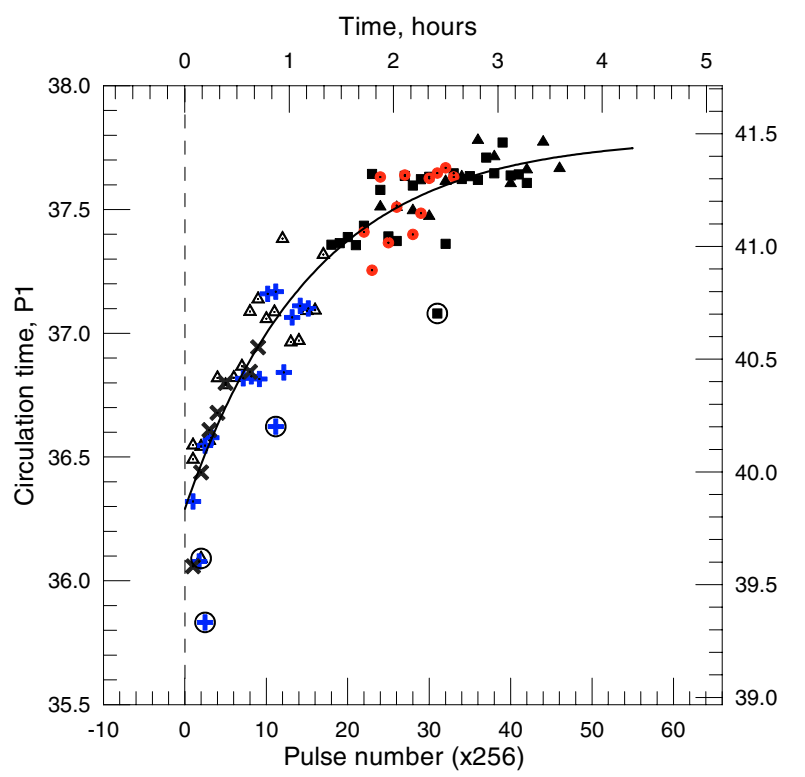

Fig. 8. Subbeam "carousel" circulation time $\hat{P}_{3}$ as a function of time from the B-mode onset at intervals of $256 P_{1} ; 1 \mathrm{~h}$ is equal to $12.8 \mathrm{such}$ units. Circulation times for the three Q- to B-mode transition observations are aligned at the origin (vertical dotted line) and fitted with the exponential curve (solid line). The other three "pure B"-mode observations could be fitted to this curve by shifting along the time axis at 18,24 and 22 (256- $P_{1}$ units), respectively. The characteristic time is some 4000 pulses or $1.22 \mathrm{~h}$. It is also about 107 circulation times. All three days with "pure B"-profiles show a gradual $\hat{P}_{3}$ increase from some 37.3 to about $37.8 P_{1}$ during the observational session and thus seem to be a later part of the Q- to-B transformation process. Four of the lrf spectra exhibited double peaks and $\hat{P}_{3}$ s calculated for these weaker precursor peaks are separately indicated (open circles) but not included in the fitting procedure (see text).

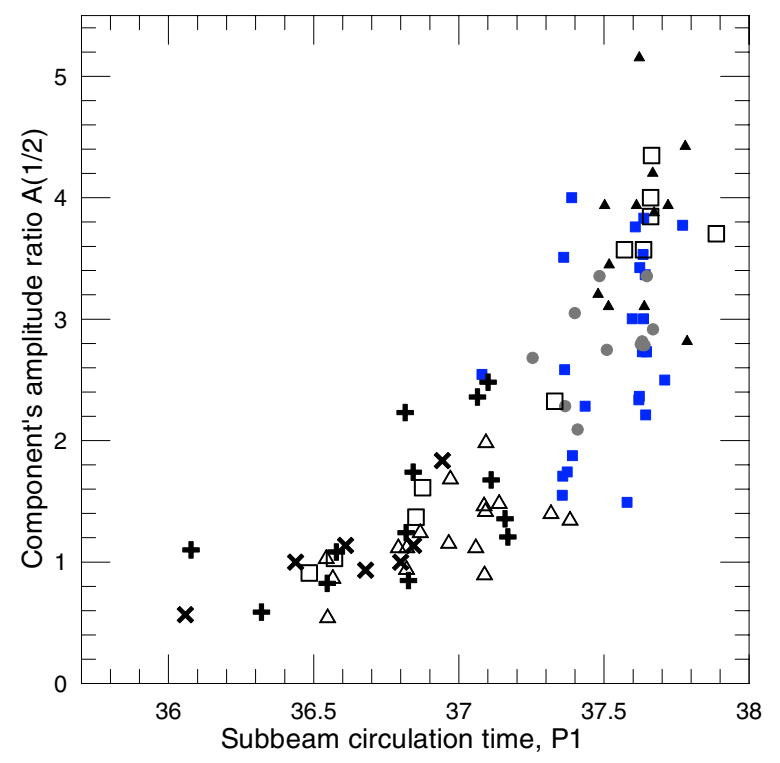

Fig. 9. Component amplitude ratio $A(1 / 2)$ as a function of subbeam circulation time $\hat{P}_{3}$ at $327 \mathrm{MHz}$. The $103-\mathrm{MHz}$ values (open square symbols) were taken from Table 1 of Paper III.

observations as well as some further observations from Paper III (open square symbols). The values are well fitted by an inverse relation. Overall, it is clear that the subbeam-circulation time and profile form are closely related, such that one could reliably be estimated from the other. 


\section{Asymmetry in the mode-changing process}

We see good evidence from the foregoing analyses that the modal-transformation process of the emitting regime in pulsar B0943+10 is not symmetrical in time. Thus far we have only managed to observe one B-to-Q-modal transition - this the remarkable 18-min (986- $P_{1}$ ) Arecibo 430-MHz observation obtained in 1992, which was thoroughly investigated in both SIRR and Paper I.

Comparison of the evolution of the pulse shape and subbeam-carousel rotation time $\hat{P}_{3}$ for the respective Q-to-B (2003) and B-to-Q (1992) transitions reveals significant differences - i.e., in the latter case these processes occur much more quickly.

To investigate the pulse-shape evolution we divided the pulse window onto two subwindows $\mathrm{W} 1$ and $\mathrm{W} 2$ at negative $\left(-8^{\circ}\right.$, $0)$ and positive longitudes $\left(0,+8^{\circ}\right)$. As was shown earlier at $430 \mathrm{MHz}$, the maxima of the B-mode and Q-mode profiles are separated in longitude at a distance nearly equal to the halfpower width of the B-mode profile. This means that a longitude on the trailing edge of the B-mode profile corresponding to the half-power point can be taken as a zero longitude, equally remote from regions where the dominance of each mode is strongest. Temporal variations of the intensity ratio in these two subwindows W1/W2 are presented in Fig. 10. The intensity of the subwindow for each individual pulse is obtained by summing the amplitudes of all the bins for a given subwindow - besides additionally averaging over 100 adjacent pulses to diminish the influence of the noise and possible scintillation fluctuations. Note that the W1/W2 ratio changes from 2.8 to 0.8 during the 18-min B-to-Q profile-shape transformation; whereas in the opposite Q-to-B transition the same change in pulse shape takes about $2.5 \mathrm{~h}$ ! Similarly, the $\hat{P}_{3}$ value at the B-mode recommencement is just over $36 P_{1}$, while at the Q-mode onset it was about $37.35 P_{1}$. One can suggest that a sharp decrease of the circulation time down to $36 P_{1}$ occurs after Q-mode commencement, without further significant changes of the pulse shape. Note that the $\mathrm{W} 1 / \mathrm{W} 2$ ratio is nearly constant (W1/W2 is randomly distributed in the range $0.6-1.0)$ during the initial 46-min (2540 pulses) Q-mode interval of 10 March and the 96-min (5266 pulses) Q-mode PS of 12 July.

\section{Discussion}

Pulsar B0943+10's circulation time $\hat{P}_{3}$ in Fig. 8 gradually changes in the range from about $36 P_{1}$ to at least $37.8 P_{1}$ following the onset of its B mode. The difference $(1.8 / 36=0.05)$ may be as large as $5 \%$.

Drift-rate variations are well known to occur in a number of pulsars, and we now see that B0943+10 is another member of this group. Some stars such as B0031-07 (e.g., Wright \& Fowler 1981a; Vivekanand \& Joshi 1997), B1944+17 (e.g., Deich et al. 1986), B2303+30 (Redman et al. 2005) and B2319+60 (e.g., Wright \& Fowler 1981b) exhibit several discrete drift-rate "states" that are usually designated as modes. Others show what appear to be continuous drift-rate changes from rarely (i.e., B0809+74; van Leeuwen et al. 2002) to very frequently (B0826-34 and B2016+28) - and in B0031-07 and $\mathrm{B} 2303+30$ a regular cycle has been detected following occasional "events" wherein "null" pulses are often observed to accompany drift-rate variations.

While the discrete drift rates can change by several factors of two, the amplitude of observed continuous drift-rate variations is less than $8 \%$ as in B0943+10, and their characteristic

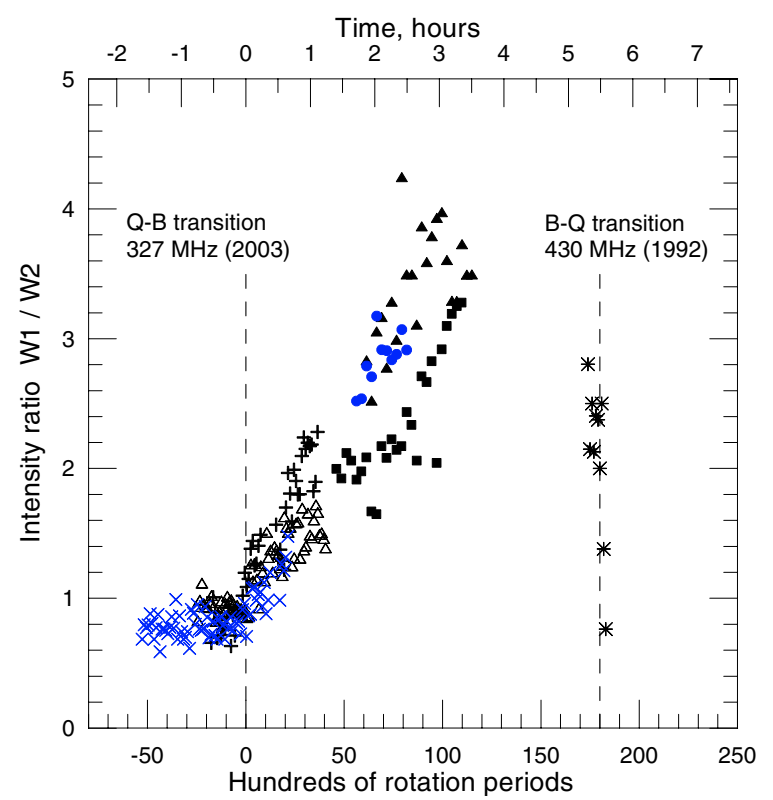

Fig. 10. Intensity ratio of two emission sub-windows for negative longitudes $\mathrm{W} 1$ and positive longitudes $\mathrm{W} 2$ as a function of time following the B-mode onset in units of the group number of successive 100-pulse averages. The pulse shape is apparently stable during the $1.5 \mathrm{~h}$ of Q-mode emission, whereas it continuously changes after the B-mode recommencement. The mode transition times are shown by vertical dashed lines. The shift of the $1992 \mathrm{~B}$ - to Q-mode observation (430 MHz) along time axis is arbitrary. This figure clearly shows the different pulse-shape transformation rate for Q-to-B- (slow) and B-to-Q-mode (fast) transitions in B0943+10.

times some several minutes - i.e., significantly less than we observe in B0943+10. Moreover, B0943+10 surely provides the best example of drift-rate changes which correlate with large changes in the profile form. In most other such pulsars driftrate variations are associated only with smaller and less orderly changes in the amplitude, shape and/or phase of the pulse profile. Systematic variations of the drift rate in B0826-34 (and probably B2303+30) apparently cross alias boundaries and thus produce changes in the drift direction (Esamdin et al. 2004; Gupta et al. 2004). An even stronger similarity with B0943+10 is the discovery by Esamdin et al. that B0826-34's long intervals of "null" pulses are in fact a weak Q-like mode, which has a very different profile from that of the strong, drifting mode (see their Fig. 2). This weak mode lasts longer than the stronger mode, and its profile is also found to be stable in intensity and shape. Moreover, both stars are now known to have closely aligned geometries.

In $\mathrm{B} 0943+10$ it is notable that null pulses have not been detected despite its prominent drift-rate variations. In its bright $\mathrm{B}$ mode an upper limit on the number of nulls might be placed at around $0.1 \%$, but in the chaotic Q mode the intensity range of individual pulses is so great that no sensible discrimination can yet be made between possible nulls and weak pulses.

An effort to understand drift-rate variations in the context of revisions to the polar-cap accelerator theory of Ruderman $\&$ Sutherland (1975) has recently been published by Gil et al. (2003). These authors attribute such variations to the shielding effect of iron ions whose flow is sensitive to the surface temperature of the polar cap, which in turn is heated by the bombardment of ultra-relativistic charged particles originating from pair production. The model implies that the cooling process of the star's surface below the mean temperature leads to a faster than mean drift rate, while the reverse process of heating 
results in slower than mean drift rates. The drift-rate and direction changes of B0826-34 at $325 \mathrm{MHz}$ were discussed by Gupta et al. (2004). A typical time scale for one cycle of drift-rate reversal is about 100 stellar rotation periods, or nearly 3 min for this pulsar. In B0826-34 drift-rate increases (corresponding to cooling below the mean temperature) are found to occur more slowly and smoothly than drift-rate decreases.

In B0943+10 the cycle is just the opposite: the slowly decreasing B-mode drift rate (corresponding ostensibly to heating above the mean temperature) is the slower process. Thus, this mechanism would appear to be backward for B0943+10, contrary to B0826-34. Nevertheless, it is clear that the circulationtime increases and decreases of the subbeam carousel have different characteristic times for the two pulsars. The current theory predicts very short cooling time scales (a few $\mu$ s for magnetic fields strengths of a few $10^{12} \mathrm{G}$; Gil et al. 2003) that could explain microstructure of individual pulses or short nulls. That any physical mechanism could cause multi-hour-scale variations in the polar cap temperature in pulsars is still very much open to question.

As mentioned above, a number of pulsars exhibit drift-rate variations in a range up to about $8 \%$ like B0943+10. None of the others, however, demonstrate B0943+10's pronounced and correlated changes in profile form. Though we cannot be sure what agent or mechanism is causing these profile variations, we are attracted by the analogy to the average pulse-shape changes in two well studied "mode switchers", B0329+54 and B1237+25. Core emission is prominent in these two stars, surely in substantial part because our sightline passes much closer to their respective magnetic axes, but in both cases clear modal variations are also seen in the characteristics of their conal components. A tendency for profile modes to represent more and less symmetrical configurations of emission (about the longitude of the magnetic axis) has long been noted (e.g., Rankin 1986), and the contrasting properties of the modes in these two stars is particularly striking in this respect: both exhibit roughly symmetrical profiles in their respective "normal" modes and then strong leading/trailing emission asymmetries accompanied by enhanced core activity in their "abnormal" modes. In B0329+54 at meter wavelengths (e.g., some 111-606 MHz), mode changes are marked by alterations in the positions and intensities of the core and leading conal components (Gangadhara \& Gupta 2001; Suleymanova \& Pugachov 2002), and a very similar pattern is observed in B1237+25 (Bartel et al. 1982; Srostlik \& Rankin 2005) as well apparently as in B0355+54 in the course of its slow modal variations (Morris et al. 1980).

In B0943+10 [as in other conal single $\left(\boldsymbol{S}_{\mathrm{d}}\right)$ stars] our sightline cuts across the periphery of its conal emission pattern, so we can have no direct indicator of its core radiation intensity. Nevertheless, we can hypothesize that B0943+10 belongs to the group of pulsars with variable core activity. In B0329+54 and $\mathrm{B} 1237+25$, core-active intervals are observed as sudden eruptions, whereas in B0943+10, this activity may increase gradually over several hours. As a result the star's symmetrical profile (seen in its Q mode and also just after B-mode onset) evolves to its usual, highly asymmetrical form several hours into each B-mode interval. It is also possible that the profile change at B-mode recommencement from "single" to "double resolved" entails a conal emission-height increase that is prompted by core activity (indeed, a possibility worth exploring for B0826-34 as well).

Recent XMM-Newton observations by Zhang et al. (2005) have detected X-ray emission from B0943+10, and several current models suggest that such radiation is basically thermal, emitted from a polar cap surface heated by back-flowing plasma produced by the same pair-processes that are responsible for the star's radio emission. These authors conclude that "within the thermal interpretation, the X-ray radiation is emitted from a heated area much smaller than the conventional polar cap area". This result, however, was based on a 20-ks observation - one surely long enough to involve one or another (or a mixture) of B and Q-mode intervals. Increased core activity (associated with one of the modes) might alter the polar-cap surface temperature and distribution (if the cooling time-scales are indeed long enough), affecting $\boldsymbol{E} \times \boldsymbol{B}$ and/or the conal beam symmetry as mentioned above. The above result takes no account of this and is thus of uncertain significance.

If, however, the "feedback" model of Wright (2003) is substantially correct, the situation may be much more complex. Here the "carousel" circulation time would depend on the "connectivity" between the polar cap and "outer gap" accelerators, and only indirectly on the $\boldsymbol{E} \times \boldsymbol{B}$ drift. In this aurora-like scenario, the variation in both rotation speed and profile asymmetry would be produced by the inevitably asymmetric interaction between the magnetic poles of an inclined rotating star. Even in a perfect dipolar geometry, the slowly circulating emission columns at either pole would not remain on matching fieldlines and their communication paths would gradually become "twisted" in a manner not unlike the earlier suggestion of Paper III.

\section{Summary and conclusions}

B0943+10's subpulse-drift rate is observed to decrease exponentially by some $5 \%$ during the $4+\mathrm{h}$ after B-mode onset with a characteristic time of some $73 \mathrm{~min}$ - and a very similar behavior is seen in all three $327-\mathrm{MHz}$ observations. These driftrate changes are accompanied by changes in the integrated pulse shape. Remarkably, the trailing component brightens at B-mode onset, making the overall profile a conal double, and for the first $20 \mathrm{~min}$ or so at $327 \mathrm{MHz}$ it outshines the usual leading emission feature. Overall, the amplitude ratio of the two B-mode profile components varies significantly from 1.75 to 0.2 during the same 4+ h. Apparently this slowly decreasing drift rate eventually falls below some critical value, and is replaced by the disorderly drift pattern of the alternative $\mathrm{Q}$ emission mode. This Q mode is characterized by a broad Gaussian-shaped profile that is stable over several hours, a complete absence of regular subpulse drift, and a diminuation to about half in emission intensity. This highly organized process of gradually decreasing subpulse drift rate accompanied by systematic profile variations has no simple parallel in other radio pulsars. In particular, no other known pulsar exhibits systematic variations on a time scale even approaching the $1.2 \mathrm{~h}$ seen in B0943+10.

Our main results can be summarized as follows:

- Six observations at $327 \mathrm{MHz}$ with a total duration of more than $11 \mathrm{~h}$ were carried out in 2003 in order to search for modal transitions.

- Three of the days exhibited Q- to B-mode transitions and three others were found to have long B-mode sequences.

- All three Q- to B-mode transitions appear to behave very similarly, qualitatively and quantitatively.

- A Q-mode circulation-time feature was detected for the first time, and its value is some $36.4 \pm 0.9 P_{1}$.

- The B-mode circulation time is found to be some $36.2 P_{1}$ just after its onset.

- The B-mode circulation time exhibits an exponential relaxation to an asymptotic value of perhaps $37.8 P_{1}$ with a characteristic time of $1.2 \mathrm{~h}$. 
- The early B-mode profile is found to be double - with a somewhat larger second component - which then gradually decreases over a several hour period, such that the magneticaxis longitude falls at the trailing half-power point of the asymptotic B-mode profile.

- The symmetrical to asymmetrical subbeam-carousel visibility - demonstrated by the dramatic B-mode profile variations - points to a complex physical origin related to the "absorption" phenomenon.

- Q-to-B-mode changes in pulsar B0943+10 again have a rapid and very slow aspect. The $1.2-\mathrm{h}$ characteristic time may be the longest orderly behavior known to be associated with pulsar emission.

Acknowledgements. We thank Geoff Wright for his critical contributions to the paper in draft. This work was also supported in part by US NSF grants AST 99-86754, 00-98685 as well as (for JMR) a visitor grant from the Nederlandse Organisatie voor Wetenschappelijk Onderzoek. Arecibo Observatory is operated by Cornell University under a cooperative agreement with the US NSF.

\section{References}

Asgekar, A. A., \& Deshpande, A. A. 2001, MNRAS, 326, 1249

Bartel, N., Morris, D., Sieber, W., \& Hankins, T. H. 1982, ApJ, 258, 776
Deich, W. T. S., Cordes, J. M., Hankins, T. H., \& Rankin, J. M. 1986, ApJ, 300, 540

Deshpande, A. A., \& Rankin, J. M. 1999, ApJ, 524, 1008

Deshpande, A. A., \& Rankin, J. M. 2001, MNRAS, 322, 438

Esamdin, A., Lyne, A. G., Graham-Smith, F., et al. 2005, MNRAS, 356, 59

Gil, J. A., Melichidze, G. I., \& Geppert, U. 2003, A\&A, 407, 315

Gupta, Y., Gil, J., Kijak, J., \& Sendyk, M. 2004, A\&A, 426, 229

Gangadhara, R. T., \& Gupta, Y. 2001, ApJ, 555, P31

van Leeuwen, A. G. L., Kouwenhoven, M. L. A., Ramachandran, R., Rankin, J. M., \& Stappers, B. W. 2002, A\&A, 387, 169

Morris, D., Sieber, W., Ferguson, D. C., \& Bartel, N. 1980, A\&A, 84, 260

Rankin, J. M. 1986, ApJ, 301, 901

Rankin, J. M., Suleymanova, S. A., \& Deshpande, A. A. 2003, MNRAS, 340, 1076

Redman, S. L., Wright, G. A. E., \& Rankin, J. M. 2005, 357, 859

Ruderman, M. A., \& Sutherland, P. G. 1975, ApJ, 196, 51

Srostlik, Z., \& Rankin, J. M. 2005, MNRAS, 362, 1121

Suleymanova, S. A., \& Izvekova, V. A. 1984, Sov. Astron., 28, 53

Suleymanova, S. A., Izvekova, V. A, Rankin, J. M., \& Rathnasree, N. 1998, JAA, 19, 1 (SIRR)

Suleymanova, S. A., \& Pugachov, V. D. 2002, Astron. Rep., 46, 34

Vivekanand, M., \& Joshi, B. C. 1997, ApJ, 477, 431

Wright, G. A. E., \& Fowler, L. A. 1981a, Pulsars, ed. W. Sieber, \& R. Wielebinski (Dordrecht: Reidel), IAU Symp., 95, 211

Wright, G. A. E., \& Fowler, L. A. 1981b, A\&A, 101, 356

Wright, G. A. E. 2003, MNRAS, 344, 1041

Zhang, B., Sanwal, D., \& Pavlov, G. 2005, ApJ, 624, 109 